\title{
Fabrication of Silk Nanofibres with Needle and Roller Electrospinning Methods
}

\author{
Nongnut Sasithorn ${ }^{1,2}$ and Lenka Martinová ${ }^{3}$ \\ ${ }^{1}$ Department of Nonwovens and Nanofibrous Materials, Faculty of Textile Engineering, Technical University of Liberec, \\ Studentská 2, 46117 Liberec, Czech Republic \\ ${ }^{2}$ Department of Textile Chemistry Technology, Faculty of Industrial Textiles and Fashion Design, \\ Rajamangala University of Technology Phra Nakhon, No. 517, Nakhonsawan Road, Bangkok 10300, Thailand \\ ${ }^{3}$ Institute for Nanomaterials, Advanced Technology and Innovation, Technical University of Liberec, Studentská 1402/2, \\ 46117 Liberec, Czech Republic
}

Correspondence should be addressed to Nongnut Sasithorn; nongnut.s@rmutp.ac.th

Received 5 May 2014; Revised 16 July 2014; Accepted 5 August 2014; Published 8 September 2014

Academic Editor: Takuya Tsuzuki

Copyright (c) 2014 N. Sasithorn and L. Martinová. This is an open access article distributed under the Creative Commons Attribution License, which permits unrestricted use, distribution, and reproduction in any medium, provided the original work is properly cited.

In this study, silk nanofibres were prepared by electrospinning from silk fibroin in a mixture of formic acid and calcium chloride. A needle and a rotating cylinder were used as fibre generators in the spinning process. The influences of the spinning electrode and spinning parameters (silk concentration and applied voltage) on the spinning process, morphology of the obtained fibres, and the production rate of the spinning process were examined. The concentration of the spinning solution influenced the diameter of the silk electrospun fibres, with an increase in the concentration increasing the diameters of the fibres in both spinning systems. The diameters of the electrospun fibres produced by roller electrospinning were greater than those produced by needle electrospinning. Moreover, increasing the concentration of the silk solution and the applied voltage in the spinning process improved the production rate in roller electrospinning but had less influence on the production rate in needle electrospinning.

\section{Introduction}

In recent years, polymer nanofibres have gained much attention as promising materials due to their unique properties, such as a high specific surface area, small pore diameters, and ability to act as a barrier against microorganisms [1-3]. They have shown enormous application potential in diverse areas, including filtration, energy storage, catalyst and enzyme carriers, drug delivery and release control systems, and tissue engineering scaffolds. There are several methods to produce fibres at the nanoscale [4]. One of these, electrospinning, has attracted a lot of interest in the last decade. Electrospinning was described as early as 1934 by Anton [5]. It is a simple but effective method to produce polymer fibres with a diameter in the range of several micrometres down to tens of nanometres, depending on the polymer and processing conditions $[4,5]$.
Electrospinning technology can be divided into two branches: conventional or needle electrospinning and needleless electrospinning. The conventional electrospinning setup normally comprises a high-voltage power supply and a syringe needle or capillary spinner connected to a power supply and a collector. During the electrospinning process, a high electric voltage is applied to the polymer solution. This leads to the formation of a strong electric field between the needle and the opposite electrode, resulting in the deformation of the solution droplet at the needle tip into a Taylor cone. When the electric force overcomes the surface tension of the polymer solution, the polymer solution is ejected off the tip of the Taylor cone to form a polymer jet. Randomly deposited dry fibres can be obtained on the collector due to the evaporation of solvent in the filament $[5,6]$. As a needle can produce only one polymer jet, needle electrospinning systems have 


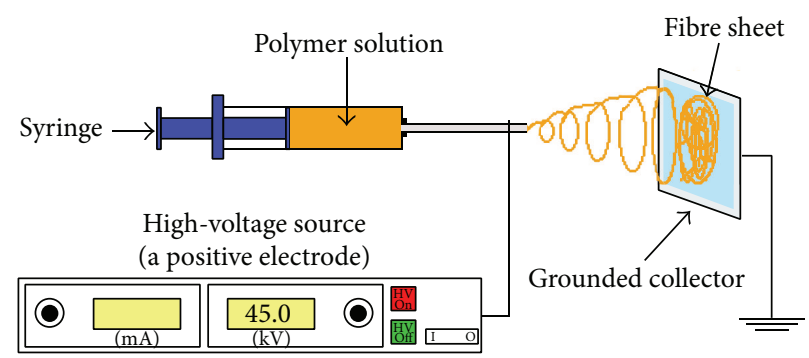

(a) Needle electrospinning

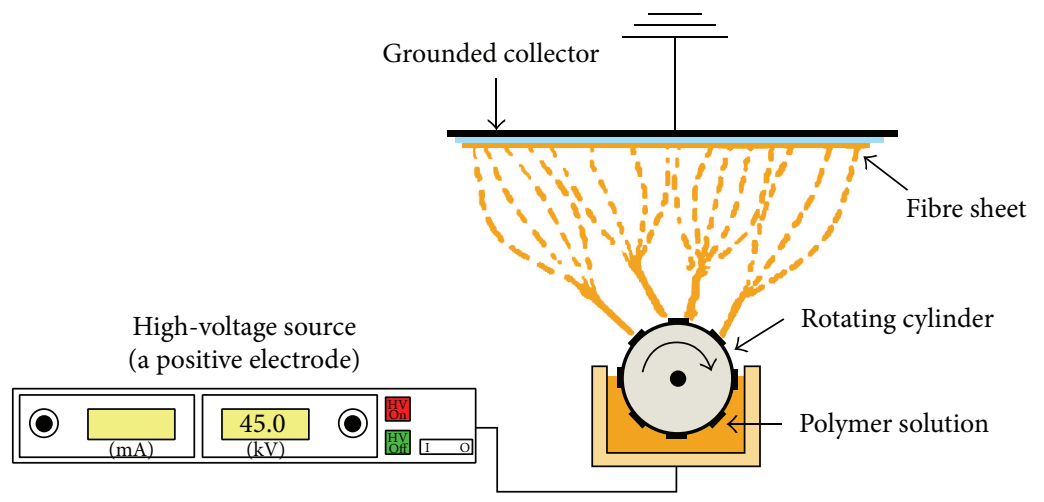

(b) Roller electrospinning

FIGURE 1: Schematic of an electrospinning experiment.

very low productivity, typically less than $0.3 \mathrm{~g} / \mathrm{h}$ per needle, making it unsuitable for practical uses [7].

Needleless electrospinning systems have been developed recently. In needleless electrospinning, instead of the generation of a polymer jet from the tip of the needle, polymer jets form from the surface of free liquid by self-organization [6-14]. For example, Jirsak et al. [9] invented a needleless electrospinning system using a roller or cylinder as the fibre generator, which was commercialized by Elmarco Co. (Czech Republic) with the brand name "Nanospider." The roller electrospinning device contains a rotating cylinder electrode, which is partially immersed in a polymer solution reservoir. When the roller slowly rotates, the polymer solution is loaded onto the upper roller surface. Upon applying a high voltage to the electrospinning system, a number of solution jets are simultaneously generated from the surface of the rotating spinning electrode, thereby improving fibre productivity [5].

Silk is a fibrous protein produced by a variety of insects, including the silkworm. Silk filament is a double strand of fibroin, which is held together by a gummy substance called silk sericin or silk gum [15]. It also contains minor amounts of residues of other amino acids and various impurities: fats, waxes, dyes, and mineral salts. Depending on the cocoon strain, the fibroin content is $66.5-73.5 \mathrm{wt} \%$, and the sericin content is $26.5-33.5 \mathrm{wt} \%$ [16]. Silk fibroin gives high mechanical strength, elasticity, and softness. In addition to its outstanding mechanical properties, it is a candidate material for biomedical applications because it has good biological compatibility and oxygen and water vapour permeability, in addition to being biodegradable and having minimal inflammatory reactions. Silk fibroin is used in various areas, such as cosmetics, medical materials for human health, and food additives [17-22]. Various forms of silk fibroin, such as gels, powders, fibres, and nonwoven membranes, can be regenerated by dissolution, followed by recovery $[15,16]$.

Natural silk fibres dissolve only in a limited number of solvents because of the presence of a large amount of intra- and intermolecular hydrogen bonds in fibroin and its high crystallinity. Consequently, hydrogen bonds have an important effect on the conformation and structure of fibroin. The influence of hydrogen bonding on the stability of fibroin molecules can be seen by the ease with which protein dissolution occurs in known hydrogen bond-breaking solvents. Silk fibroin can be dissolved in concentrated aqueous solutions of acids $\left(\mathrm{H}_{3} \mathrm{PO}_{4}, \mathrm{HCOOH}, \mathrm{H}_{2} \mathrm{SO}_{4}\right.$, and $\left.\mathrm{HCl}\right)$ and in high ionic strength aqueous salt solutions, such as lithium bromide ( $\mathrm{LiBr})$, calcium chloride $\left(\mathrm{CaCl}_{2}\right)$, and magnesium chloride $\left(\mathrm{MgCl}_{2}\right)$. The main disadvantage of a salt-containing aqueous solvent is the long preparation time because aqueous solutions of fibroin have to be dialyzed for several days to remove the salts and to recover the polymer as films, sponges, or powder from the aqueous solution by dry forming. In some organic solvents (e.g., hexafluoroisopropanol and hexafluoroacetone), fibroin can be dissolved only after preliminary activation by dissolution in aqueous salt systems [15, 23]. Previous studies showed that silk fibroin can be dissolved in a mixture of formic acid and calcium chloride and form into films or it can be spun into nanofibres by the electrospinning method $[24,25]$.

In the present study, we investigated the fabrication of silk electrospun fibres with two different spinning systems: a needle and a roller, concentrating on the effect of the spinning 

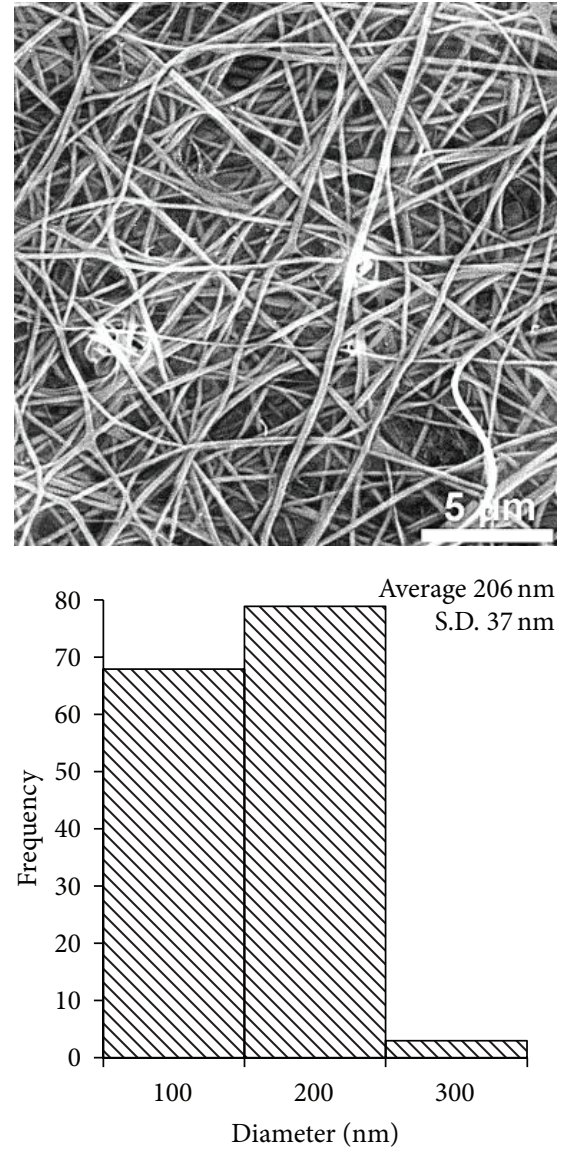

(a)
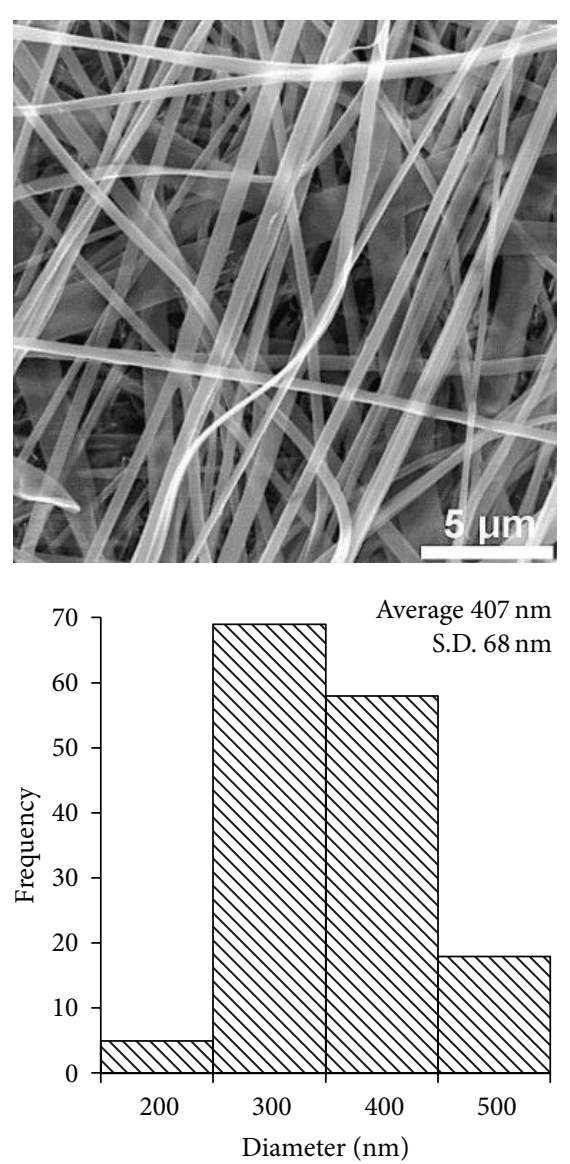

(b)
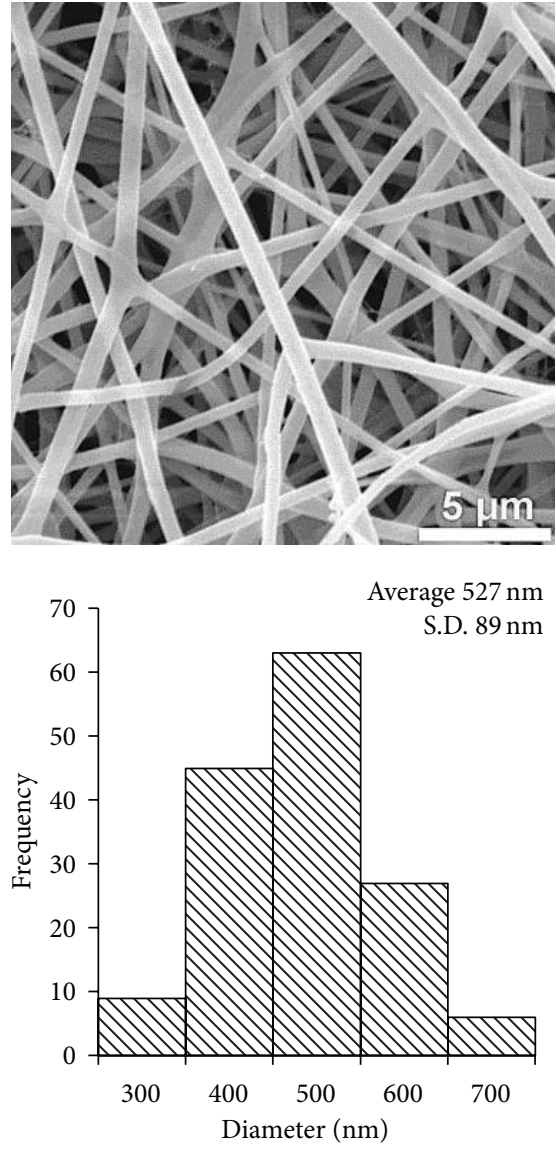

(c)

FIGURE 2: SEM micrographs and fibre distribution of electrospun fibres produced by needle electrospinning with silk fibroin solution at various concentrations. (a) $8 \mathrm{wt} \%$; (b) $10 \mathrm{wt} \%$; (c) $12 \mathrm{wt} \%$.

electrode on the electrospinning process. We also studied the influences of the concentration of the spinning solution and applied voltage on the morphology of the obtained electrospun fibres and the production rate of the spinning process.

\section{Experiment}

2.1. Materials. Thai silk cocoons of Bombyx mori Linn. silkworms (Nang-Noi Srisakate 1) were obtained from Amphoe Mueang Chan, Si Sa Ket Province, Thailand. ECE Phosphate Reference Detergent FBA free (Union TSL Co., Ltd., Thailand) was used as a soaping agent in the degumming process. The chemicals used for the preparation of the spinning solutions were calcium chloride (Fluka AG, Switzerland) and 98\% formic acid (Penta, Czech Republic). All other chemicals used in this study were reagent grade.

2.2. Preparation of Silk Fibroin Solutions. Raw silk cocoons were degummed twice with $0.1 \mathrm{M}$ of sodium carbonate and $0.5 \%$ of standard reference detergent at $100^{\circ} \mathrm{C}$ for $30 \mathrm{~min}$, rinsed with warm water to remove the sericin from the surface of the fibre, and then dried at room temperature. Silk fibroin solutions were prepared by dissolving the degummed silk fibres in a mixture of formic acid (98\%) and calcium chloride. The ratio of silk fibre to calcium chloride was $1: 0.25$ $(\mathrm{w} / \mathrm{w})$. The silk fibroin concentration varied from 8 to $12 \mathrm{wt} \%$. All solutions were magnetically stirred at room temperature overnight.

\subsection{Electrospinning. A schematic representation of the} equipment used in the experiment is illustrated in Figure 1. During the needle electrospinning, the silk fibroin solution was placed in a $10 \mathrm{~mL}$ syringe with a stainless steel needle, which was connected to a high-voltage DC power supply (Spellman SL150). The flow rate of the spinning solution was $1.5 \mathrm{~mL} / \mathrm{h}$ using a syringe pump (KDS $100 \mathrm{CE}, \mathrm{KD}$ Scientific Inc., USA). The syringe used in the experiment had an 18-gauge needle (capillary diameter $1.2 \mathrm{~mm}$ ). The roller electrospinning device contains a rotating cylinder, $85 \mathrm{~mm}$ in length and $15 \mathrm{~mm}$ in diameter, and a solution reservoir. The solution reservoir, which has a high voltage connected to the bottom of the solution bath, was filled with the silk fibroin solution. The rotating cylinder was then partially immersed in the solution. During electrospinning, the spinning solution 

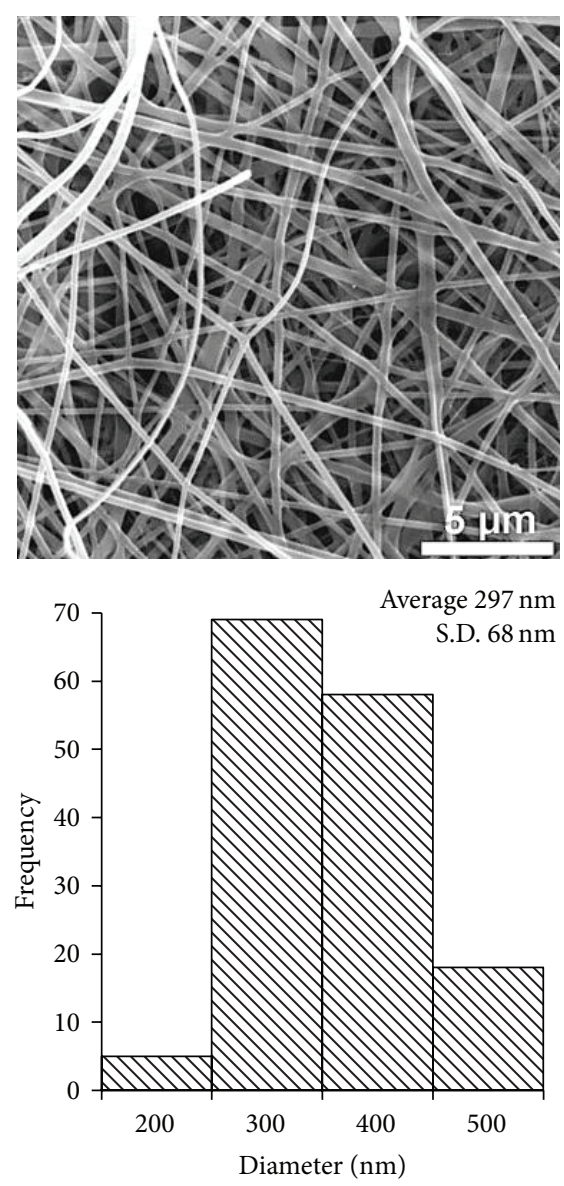

(a)
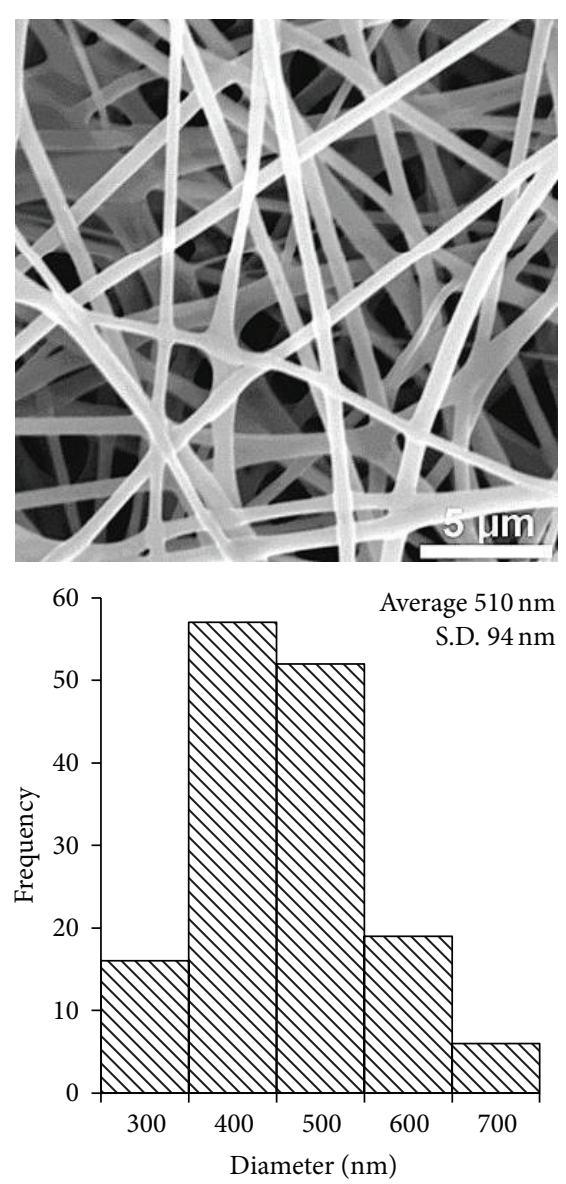

(b)
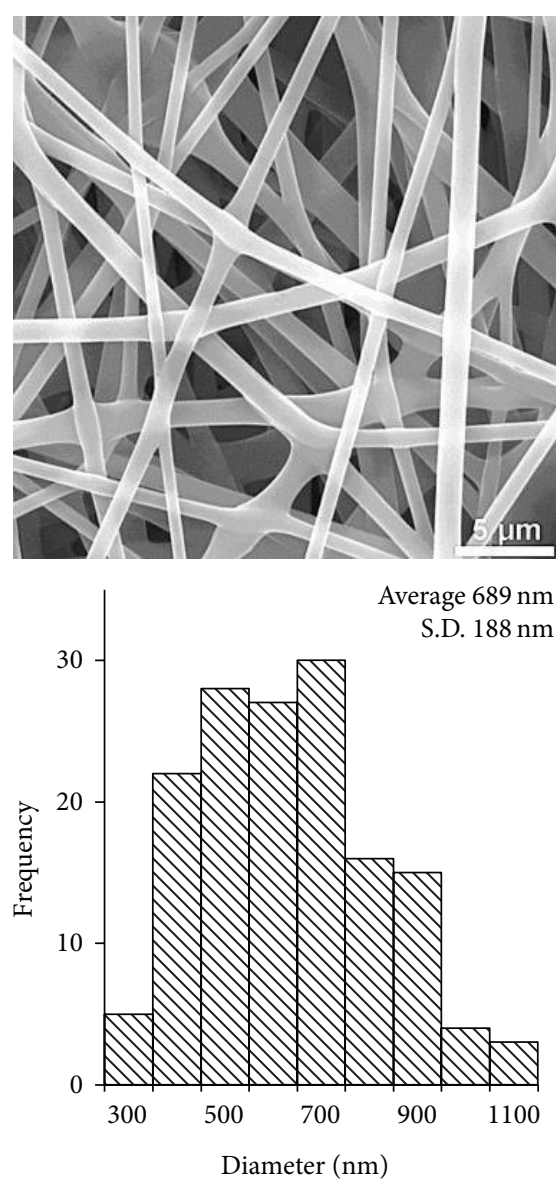

(c)

FIGURE 3: SEM micrographs and fibre distribution of electrospun fibres produced by roller electrospinning with silk fibroin solution at various concentrations. (a) $8 \mathrm{wt} \%$; (b) $10 \mathrm{wt} \%$; (c) $12 \mathrm{wt} \%$.

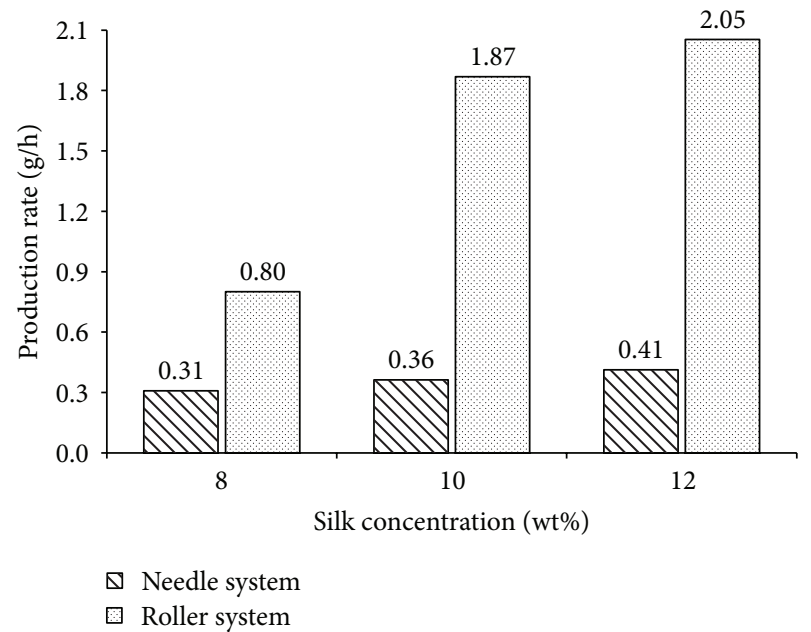

FIGURE 4: Effects of silk fibroin concentration on production rate.

was slowly loaded onto the roller surface (rotation $\sim 7 \mathrm{rpm}$ ). Electrospinning of the silk fibroin solutions was carried out at a high voltage in the range of $35 \mathrm{kV}$ to $50 \mathrm{kV}$, and the electrospun fibres were collected on a collector, which was placed at a distance of $100 \mathrm{~mm}$ from the spinning electrode. All the processes were carried out at $22^{\circ} \mathrm{C}$ and $35 \%$ humidity.

2.4. Solvent Treatments. The electrospun fibre sheets were immersed in ethanol for $30 \mathrm{~min}$ to induce crystallisation of the silk fibroin and reduce the water solubility of the fibre sheets. After drying at room temperature, the treated fibre sheets were immersed in distilled water overnight, followed by rinsing in distilled water to remove residual salts and then air-dried.

2.5. Characterisation. The viscosity of the spinning solutions was measured by a HAKKE RotoVisco RV1 rheometer (Thermo Scientific, USA). The morphological appearance of the silk electrospun fibres was observed with a scanning electron microscope (SEM) Vega 3 (Tescan, Czech Republic) at an accelerated voltage of $20 \mathrm{kV}$ under magnification of $10.0 \mathrm{kx}$. All the samples were sputter-coated (Q150R ES, Quorum Technologies Ltd., England) with gold at a thickness of $5 \mathrm{~nm}$. The SEM images were analysed with NIS-Elements AR software. The average fibre diameter and its distribution were 


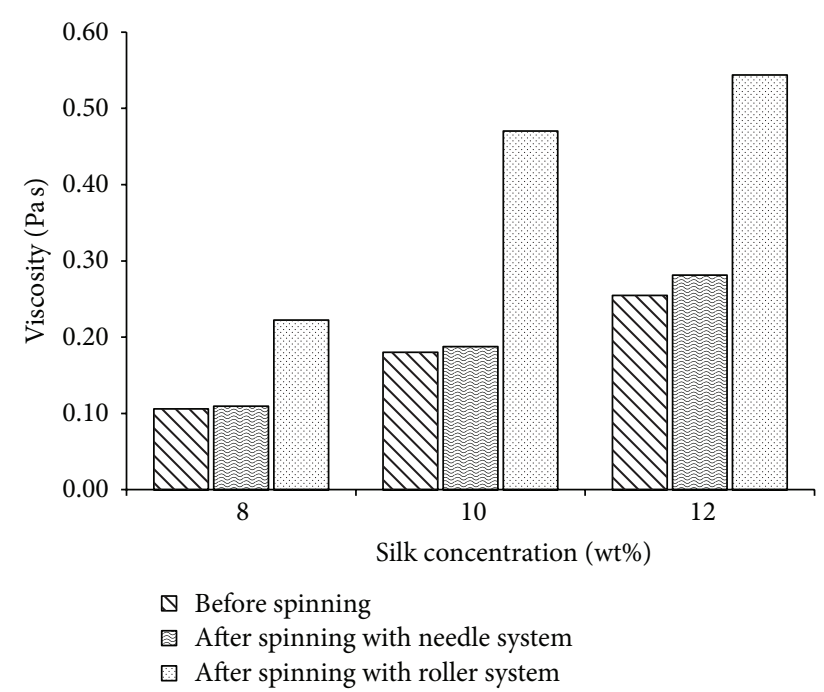

Figure 5: Comparison of the viscosity of silk fibroin solution at various concentrations.

determined from 150 random fibres obtained under each spinning condition. The production rates of the electrospun fibres with both spinning techniques were determined based on the mass of the obtained electrospun fibre sheet per unit time (the size of the samples was $10 \mathrm{~cm} \times 10 \mathrm{~cm}$ ) and then normalised to obtain the fabrication rate in grams per hour (g/h).

\section{Results and Discussion}

3.1. Effect of Silk Fibroin Concentration. The concentration of a spinning solution generally has a dominant effect on the electrospinning process. Considered the effect of concentration of spinning solution on fibre morphology with the applied voltage of $50 \mathrm{kV}$, when the silk fibroin concentration increased from $8 \mathrm{wt} \%$ to $12 \mathrm{wt} \%$. The SEM micrographs and diameter distribution of the silk fibroin electrospun fibres produced by the needle and roller electrospinning techniques are shown in Figures 2 and 3, respectively. The results show that under the same electrospinning conditions, the fibre diameter and fibre diameter distribution of the obtained electrospun fibres increased with both systems in accordance with an increase in the silk fibroin concentration, demonstrating the important role of the concentration of the spinning solution in fibre formation during the electrospinning process. The concentration of the polymer solution reflects the number of entanglements of polymer chains in the solution, which, in turn, affect the viscosity of the solution. An increase in the concentration of the silk solution will result in greater polymer chain entanglement in the solution. Thus, the viscosity of the solution also increases. At higher concentrations, the diameter of the fibre is greater. The interaction between the solution and the charges on the jet determines the distribution of the fibre diameters obtained. This is probably due to the number of jets that form during electrospinning. Multiple jets may form from the main electrospinning jet, which is stable enough to yield fibres of smaller diameter at certain concentrations, thereby generating fibres with various diameters [26, 27].

In needle electrospinning, a solution with a low concentration leads to nanofibres with beads because of the low relation of viscosity to surface tension. On the other hand, a solution with a high concentration produces fibres with greater diameters due to the limited deformability of the polymer jet and/or the shorter time needed for the solidification of the more concentrated solution $[26,28]$. In the present study, the concentration of the silk solution played an important role in the spinnability of the roller system. At low concentrations of the spinning solution, nonfibrous formations were produced instead of nanofibres with beads. It is possible that Taylor cones are created in roller electrospinning by picking up the spinning solution covering the surrounding spinning electrode $[5,14]$. Generally, in spinning solutions with a low concentration, the viscosity of the solution is also low. Such solutions cannot be loaded on the surface of the roller because of their lack of viscosity. When Taylor cones do not form on the surface of a roller, the electrospinning process results in nonfibrous formations.

In addition to affecting the fibre morphology and spinnability, the concentration of the spinning solution also influenced the production rate. The production rate of the silk electrospun fibres from roller electrospinning increased from $0.80 \mathrm{~g} / \mathrm{h}$ to $2.05 \mathrm{~g} / \mathrm{h}$ when the concentration increased from $8 \mathrm{wt} \%$ to $12 \mathrm{wt} \%$. The increase in the production rate with the needle system was less significant when the concentration of the silk solution increased (see Figure 4).

3.2. Effect of Spinning Electrode. The SEM micrographs of the silk fibroin electrospun fibres produced by the needle and roller electrospinning methods are shown in Figures 2 and 3 , respectively. The results show that under the same operating conditions, both electrospinning systems produced uniform fibres. However, the diameters of the silk electrospun fibres obtained from the needle electrospinning were smaller and the fibre diameter distribution was narrower than those obtained from the roller electrospinning. When the concentration increased from $8 \mathrm{wt} \%$ to $12 \mathrm{wt} \%$, the average fibre diameter increased from $206 \mathrm{~nm}$ to $527 \mathrm{~nm}$, respectively, in the needle system and from $297 \mathrm{~nm}$ to $689 \mathrm{~nm}$, respectively, in the roller system. It is possible that the setup of the solution bath in the roller electrospinning system, which is normally exposed to air, may increase the evaporation of solvent from the spinning solution during the spinning process. Thus, the evaporation rate of solvent in the roller system was higher than that in the needle system. The evaporation of solvent from solution can increase the concentration of a solution. As shown in Figure 5, more concentrated silk solutions increased the viscosity of the spinning solution, and the viscosity of the spinning solution was significantly higher after electrospinning with the roller system. The concentration of the spinning solution has a dominant effect on the fibre diameter, with higher concentrations generally yielding electrospun fibres with larger average diameters $[5,26]$.

In addition, Niu et al. [7] described the electric field intensity profile of a cylinder-spinning electrode in upward 

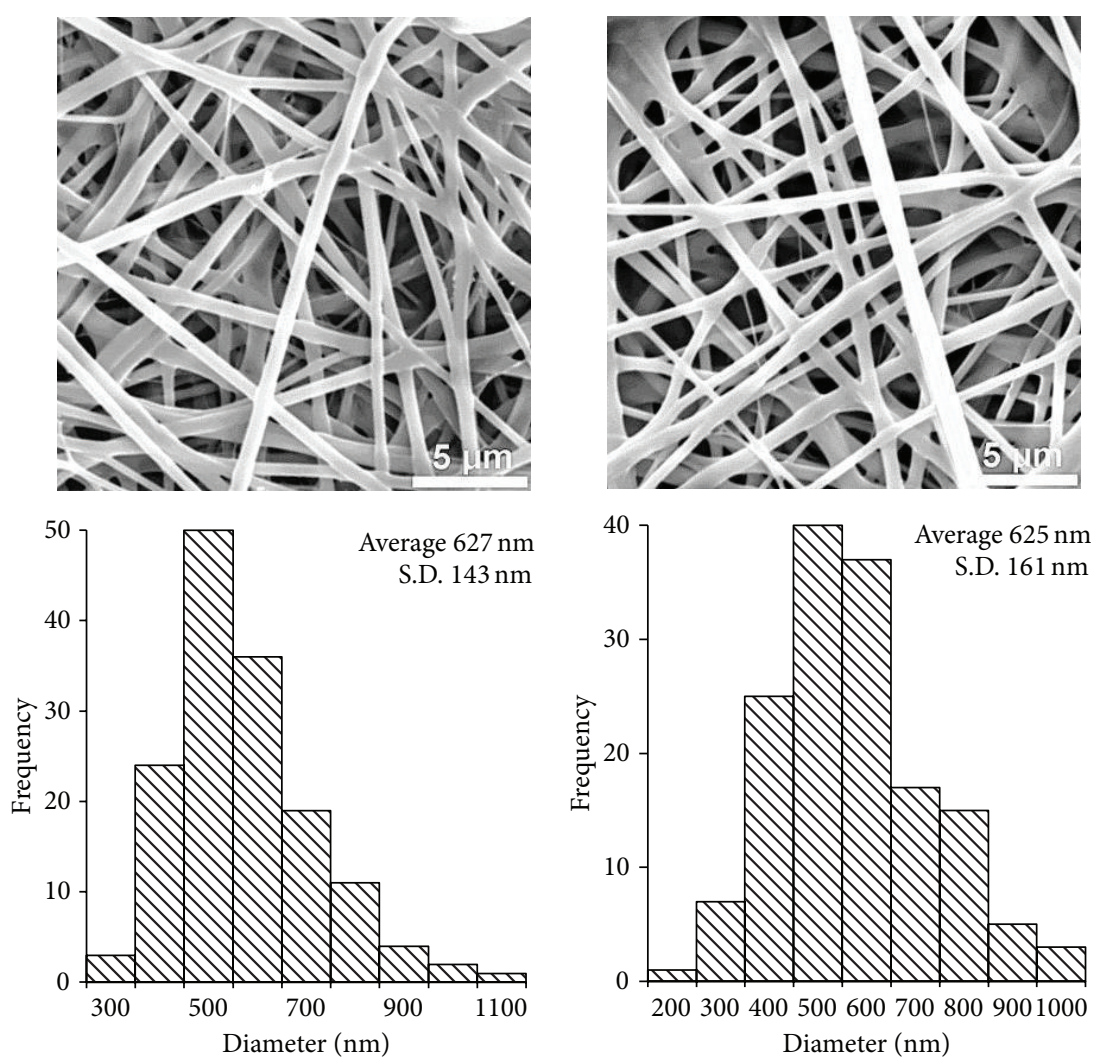

(a)
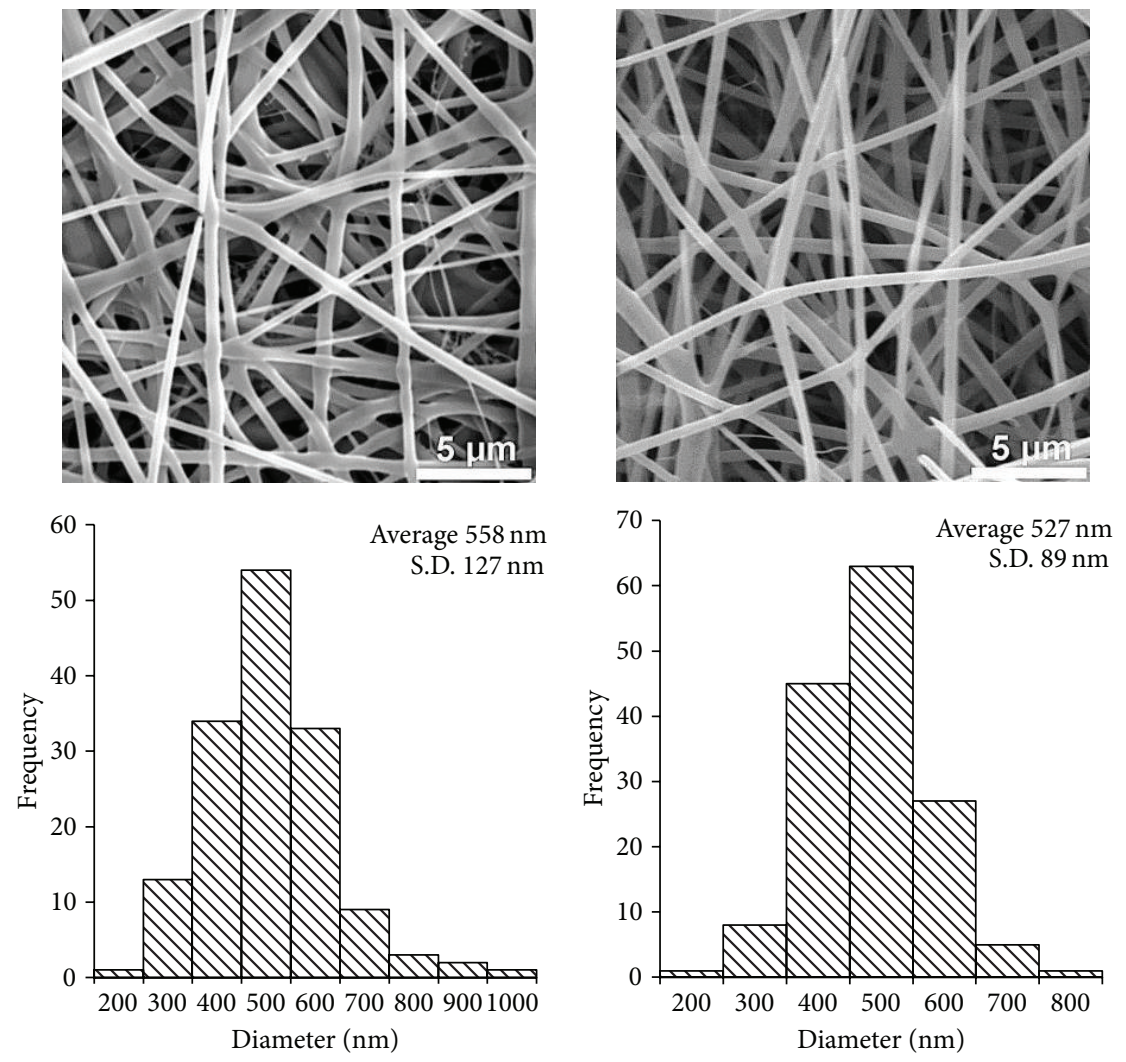

(c)

(d)

FIGURE 6: SEM micrographs of silk electrospun fibres prepared by needle electrospinning from silk fibroin (12 wt\%) at (a) $35 \mathrm{kV}$, (b) $40 \mathrm{kV}$, (c) $45 \mathrm{kV}$, and (d) $50 \mathrm{kV}$. 

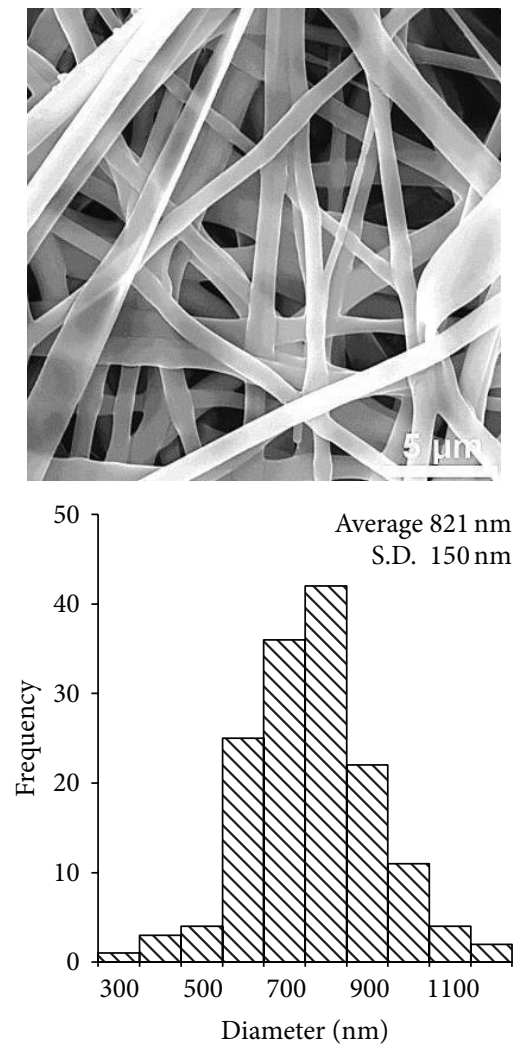

(a)
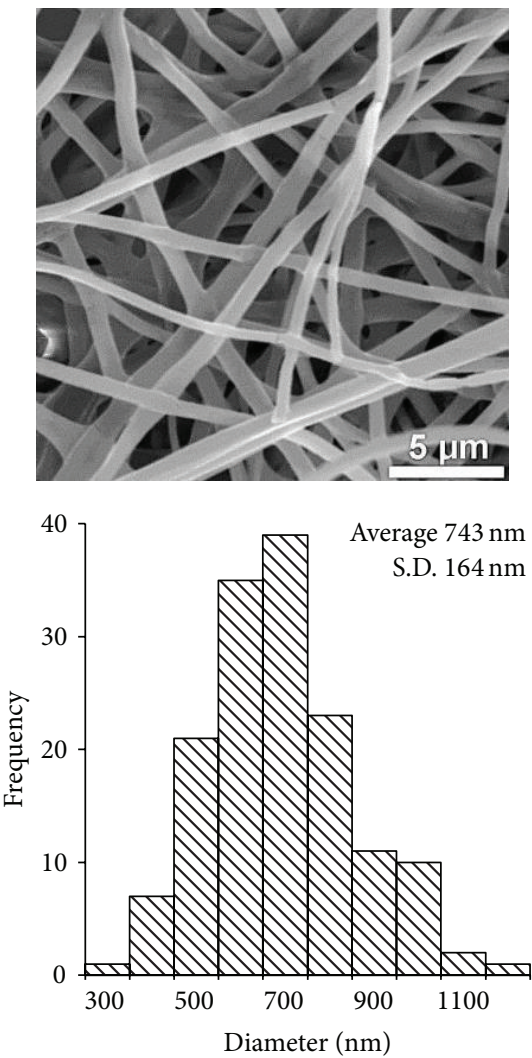

(c)
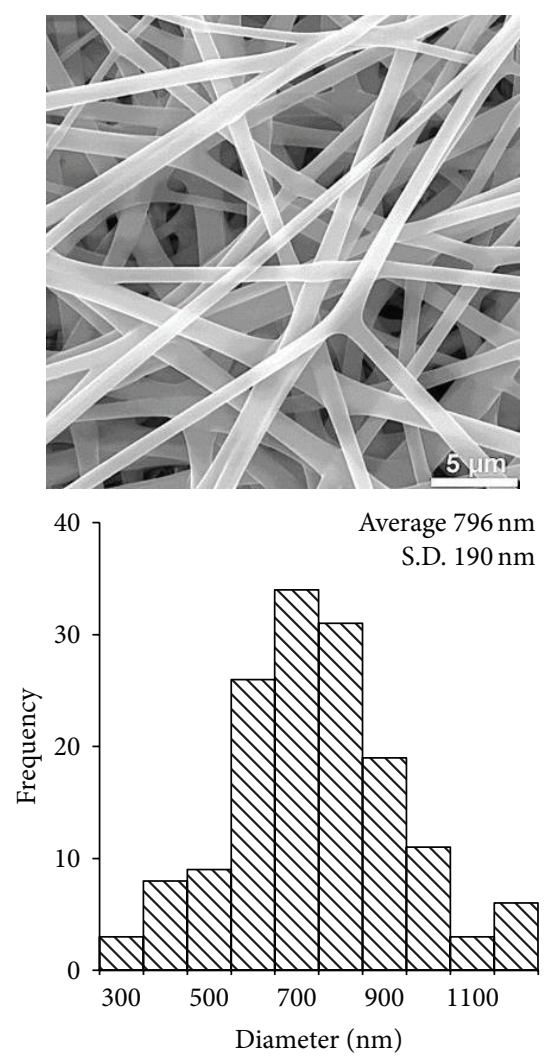

(b)
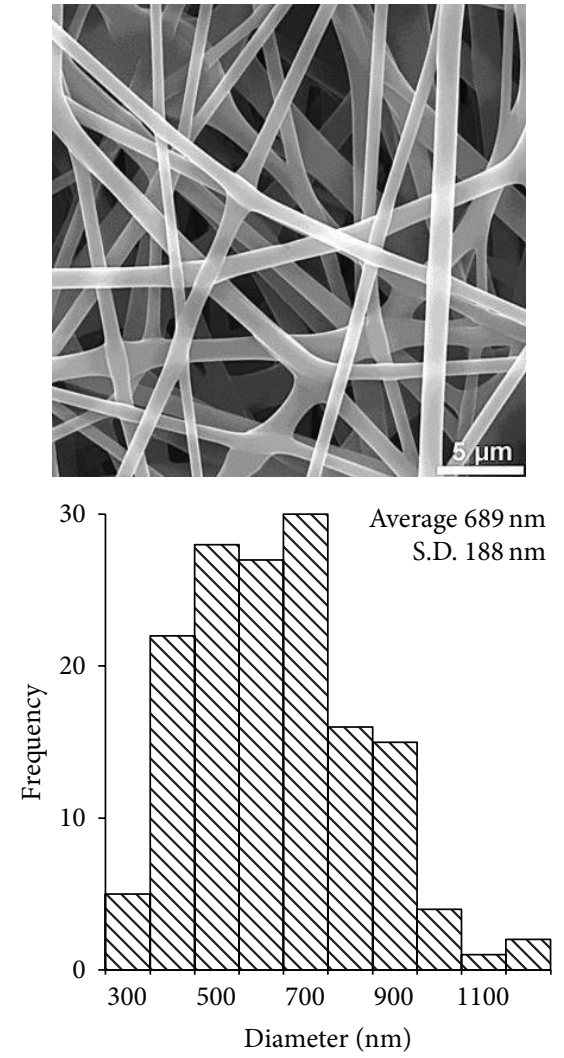

(d)

FIGURE 7: SEM micrographs of silk electrospun fibres prepared by roller electrospinning from silk fibroin (12 wt\%) at (a) $35 \mathrm{kV}$, (b) $40 \mathrm{kV}$, (c) $45 \mathrm{kV}$, and (d) $50 \mathrm{kV}$. 


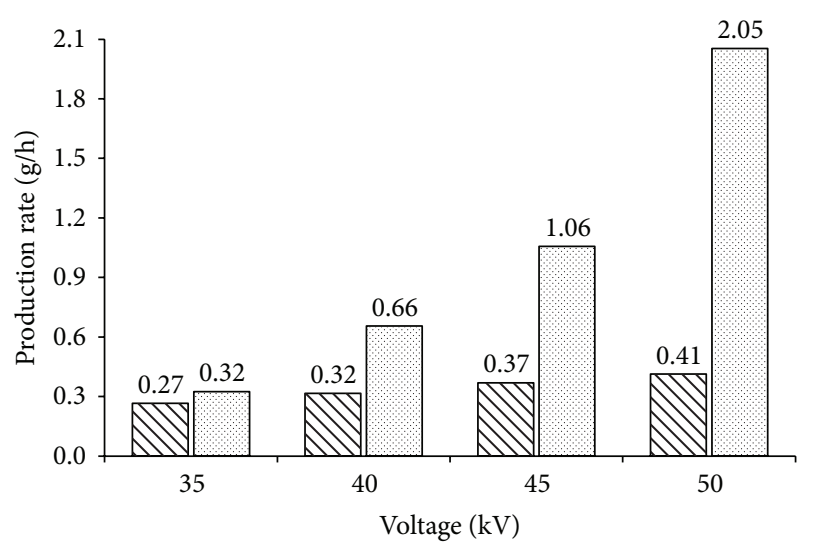

$\triangle$ Needle system

ㅁoller system

FIGURE 8: Effects of applied voltage on the production rate.

needleless electrospinning that can be used to understand electrospinning behaviours. In a rotating cylinder electrode, they observed that the electric field intensity profile was unevenly distributed along the surface of the cylinder. A high-intensity electric field mainly formed on the cylinder ends and a much lower intensity electric field formed on the middle surface area of the cylinder. Electrospinning occurred in both areas of high-electric field intensity and low-electric field intensity. Furthermore, the diameters of the electrospun fibres produced from the two areas on the roller were very different [7]. As a result, the diameter and diameter distribution of electrospun fibres from a roller system are greater than those from a needle system.

The spinning electrode affected the production rate of the electrospinning process, resulting in a higher production rate in the roller than the needle system. For example, using a $12 \mathrm{wt} \%$ silk solution and the rotating spinning electrode instead of the needle, the production rate of silk electrospun fibres increased from $0.41 \mathrm{~g} / \mathrm{h}$ to $2.05 \mathrm{~g} / \mathrm{h}$. In contrast to conventional needle electrospinning in which a Taylor cone is generated and stabilised through constantly feeding the polymer solution through the needle, a number of jets can be simultaneously generated from the layer of solution on the surface of the rotating spinning electrode in the roller electrospinning system [5]. As a result, the production rate of the roller system is much higher than that of the needle system.

3.3. Effect of Applied Voltage. To study the effect of the applied voltage on the morphology of the obtained fibres and the spinning performance, a spinning solution with a concentration of $12 \mathrm{wt} \%$ was electrospun at a voltage between $35 \mathrm{kV}$ and $50 \mathrm{kV}$. SEM micrographs of the resulting fibres and their distributions at the different applied voltages are shown in Figures 6 and 7, respectively. The applied voltage is a very important parameter with regard to the formation of jets in electrospinning systems because a high voltage is used to create an electrically charged jet of a polymer solution. Using the same polymer solution, the electric voltage required to initiate the spinning process from the roller was higher than that needed to generate fibres from the needle $[10,13]$.

In the roller electrospinning system, when the silk fibroin solution was charged with an electric voltage higher than $30 \mathrm{kV}$, a number of jets were generated from the surface of the spinning electrode. Increasing the applied voltage influenced the electrospinning process, with the average fibre diameter decreasing from $822 \mathrm{~nm}$ to $689 \mathrm{~nm}$ with an increase in the electric voltage from $35 \mathrm{kV}$ to $50 \mathrm{kV}$, respectively.

Furthermore, increasing the applied voltage affected the production rate of the spinning process. The production rate of the silk electrospun fibres from roller electrospinning changed from $0.32 \mathrm{~g} / \mathrm{h}$ to $2.05 \mathrm{~g} / \mathrm{h}$ when the applied voltage was increased (Figure 8). As the electric field is the main driving force initiating the formation of Taylor cones and jets from the surface of silk solution, increasing the electric voltage increases the electrostatic force on the polymer jet, which favours more elongation of the jet and the formation of smaller fibres. On the other hand, it is easier to generate solution jets at higher applied voltage in a polymer solution charged by a stronger electric field because a larger amount of solution is removed from the surface of the solution, thereby improving the production rate of the spinning process. Other studies also reported a tendency for decreased fibre diameters and increased production rates in different polymer systems with an increase in the applied voltage $[7,10,12]$.

In the present study, the critical voltage required to initiate nanofibres on the needle electrospinning system was lower than on the roller system. The lowest voltage for initiating a jet from the tip of the needle was $6 \mathrm{kV}$. The average fibre diameter under the operating voltage range is shown in Figure 7. Increasing the applied voltage slightly reduced the average fibre diameter. Increasing the applied voltage from $35 \mathrm{kV}$ to $50 \mathrm{kV}$ decreased the average fibre diameter from $627 \mathrm{~nm}$ to $527 \mathrm{~nm}$, respectively. Figure 8 depicts the effect of the variation in the applied electric fields on the production rate of nanofibres with the needle system. It can be seen that the effect of the applied electric field with the needle system was not as strong as with the roller system. The rate of production with the needle electrospinning system was $0.27 \mathrm{~g} / \mathrm{h}$ and $0.41 \mathrm{~g} / \mathrm{h}$ at applied voltages of $35 \mathrm{kV}$ and $50 \mathrm{kV}$, respectively.

\section{Conclusion}

We prepared silk electrospun fibre sheets using needle and roller electrospinning techniques and investigated the effect of the concentration of the silk solution, applied voltage, and spinning electrode on the morphology of the obtained fibres and the production rate of the electrospinning process. Increasing the concentration of the silk solution improved the spinning ability and the spinning performance in roller electrospinning. The concentration of the silk fibroin solution affected the fibre diameter in both spinning techniques. The silk fibre production rate of the roller electrospinning system was much higher than that of the needle electrospinning system. However, the diameter of the electrospun fibres produced with the needle system was smaller and the fibres had 
a narrower distribution than those obtained with the roller system. The applied voltage also influenced the spinning process in roller electrospinning, with an increase in the applied voltage enhancing the fibre production rate. The increase was less significant in the needle system at different applied voltages. The results suggest that roller electrospinning can improve the production rate of silk nanofibres.

\section{Conflict of Interests}

The authors declare that there is no conflict of interests regarding the publication of this paper.

\section{Acknowledgments}

This work was supported by the Technical University of Liberec, Faculty of Textile Engineering, Czech Republic, and the Ministry of Education, Youth and Sports as part of Project LO1201 targeted support from the "Národní program udržitelnosti I" Programme. The authors also thank Rajamangala University of Technology Phra Nakhon, Thailand, for providing the first author with a scholarship.

\section{References}

[1] R. M. Nerem and A. Sambanis, "From biology to biological substitutes," Tissue Engineering, vol. 1, pp. 3-13, 2007.

[2] J. J. Stankus, L. Soletti, K. Fujimoto, Y. Hong, D. A. Vorp, and W. R. Wagner, "Fabrication of cell microintegrated blood vessel constructs through electrohydrodynamic atomization," Biomaterials, vol. 28, no. 17, pp. 2738-2746, 2007.

[3] N. Amiraliyan, M. Nouri, and M. H. Kish, "Effects of some electrospinning parameters on morphology of Natural silkbased nanofibers," Journal of Applied Polymer Science, vol. 113, no. 1, pp. 226-234, 2009.

[4] T. Lin and X. Wang, Needleless Electrospinning of NanofibersTechnology and Applications, Pan Stanford Publishing Pte., 2014.

[5] H. Niu, X. Wang, and T. Lin, Needleless Electrospinning: Developments and Performances, 2012, http://cdn.intechopen.com/ pdfs/23290/InTech-Needleless_electrospinning_\%20developments_and_performances.pdf.

[6] D. Lukáš, A. Sarkar, L. Martinová et al., "Physical principles of electrospinning (Electrospinning as a nano-scale technology of the twenty-first century)," Textile Progress, vol. 41, no. 2, pp. 59$140,2009$.

[7] H. Niu, T. Lin, and X. Wang, "Needleless electrospinning. I. A comparison of cylinder and disk nozzles," Journal of Applied Polymer Science, vol. 114, no. 6, pp. 3524-3530, 2009.

[8] A. L. Yarin and E. Zussman, "Upward needleless electrospinning of multiple nanofibers," Polymer, vol. 45, no. 9, pp. 29772980, 2004.

[9] O. Jirsak, F. Sanetrnik, D. Lukas, V. Kotek, L. Martinova, and J. Chaloupek, "Method of nanofibres production from a polymer solution using electrostatic spinning and a device for carrying out the method," U.S. Patent 0290031 A1, 2006.

[10] X. Wang, H. Niu, T. Lin, and X. Wang, "Needleless electrospinning of nanofibers with a conical wire coil," Polymer Engineering and Science, vol. 49, no. 8, pp. 1582-1586, 2009.
[11] O. Jirsak, P. Sysel, F. Sanetrnik, J. Hruza, and J. Chaloupek, "Polyamic acid nanofibers produced by needleless electrospinning," Journal of Nanomaterials, vol. 2010, Article ID 842831, 6 pages, 2010.

[12] C. Huang, H. Niu, J. Wu, Q. Ke, X. Mo, and T. Lin, "Needleless electrospinning of polystyrene fibers with an oriented surface line texture," Journal of Nanomaterials, vol. 2012, Article ID 473872, 7 pages, 2012.

[13] X. Wang, H. Niu, and T. Lin, "Needleless electrospinning of uniform nanofibers using spiral coil spinnerets," Journal of Nanomaterials, vol. 2012, Article ID 785920, 9 pages, 2012.

[14] F. Cengiz and O. Jirsak, "The effect of salt on the roller electrospinning of polyurethane nanofibers," Fibers and Polymers, vol. 10, no. 2, pp. 177-184, 2009.

[15] A. Matsumoto, H. J. Kim, I. Y. Tsai, X. Wang, P. Cebe, and D. L. Kaplan, "Silk," in Handbook of Fiber Chemistry, pp. 383-404, CRC Press Taylor \& Francis Group, 2007.

[16] E. S. Sashina, A. M. Bochek, N. P. Novoselov, and D. A. Kirichenko, "Structure and solubility of natural silk fibroin," Russian Journal of Applied Chemistry, vol. 79, no. 6, pp. 869-876, 2006.

[17] C. Vepari and D. L. Kaplan, "Silk as a biomaterial," Progress in Polymer Science, vol. 32, no. 8-9, pp. 991-1007, 2007.

[18] B.-M. Min, G. Lee, S. H. Kim, Y. S. Nam, T. S. Lee, and W. H. Park, "Electrospinning of silk fibroin nanofibers and its effect on the adhesion and spreading of normal human keratinocytes and fibroblasts in vitro," Biomaterials, vol. 25, no. 7-8, pp. 12891297, 2004.

[19] N. Amiralian, M. Nouri, and M. H. Kish, "An experimental study on electrospinning of silk fibroin," 2011, http://www .docstoc.com/docs/26292086.

[20] N. Minoura, M. Tsukada, and M. Nagura, "Fine structure and oxygen permeability of silk fibroin membrane treated with methanol," Polymer, vol. 31, no. 2, pp. 265-269, 1990.

[21] Y. Wang, H.-J. Kim, G. Vunjak-Novakovic, and D. L. Kaplan, "Stem cell-based tissue engineering with silk biomaterials," Biomaterials, vol. 27, no. 36, pp. 6064-6082, 2006.

[22] B.-M. Min, L. Jeong, K. Y. Lee, and W. H. Park, "Regenerated silk fibroin nanofibers: water vapor-induced structural changes and their effects on the behavior of normal human cells," Macromolecular Bioscience, vol. 6, no. 4, pp. 285-292, 2006.

[23] G. Freddi, G. Pessina, and M. Tsukada, "Swelling and dissolution of silk fibroin (Bombyx mori) in N-methyl morpholine Noxide," International Journal of Biological Macromolecules, vol. 24, no. 2-3, pp. 251-263, 1999.

[24] U. Armato, I. D. Pra, C. Migliaresi, A. Motta, and K. Kesenci, "Method for the preparation of a non-woven silk fibroin fabrics," US Patent 7285637, 2007.

[25] N. Sasithorn and L. Martinová, "Effect of calcium chloride on electrospinning of silk fibroin nanofibres," in Proceedings of the 4th RMUTP International Conference: Textiles \& Fashion, pp. 51-58, 2012.

[26] S. Ramakrishna, K. Fujihara, W. E. Teo, T. C. Lim, and Z. Ma, An Introduction to Electrospinning and Nanofibers, World Scientific, Singapore.

[27] A. L. Andrady, Science and Technology of Polymer Nanofibers, John Wiley \& Sons, New York, NY, USA, 2008.

[28] F. Yener and O. Jirsak, "Comparison between the needle and roller electrospinning of polyvinylbutyral," Journal of Nanomaterials, vol. 2012, Article ID 839317, 6 pages, 2012. 

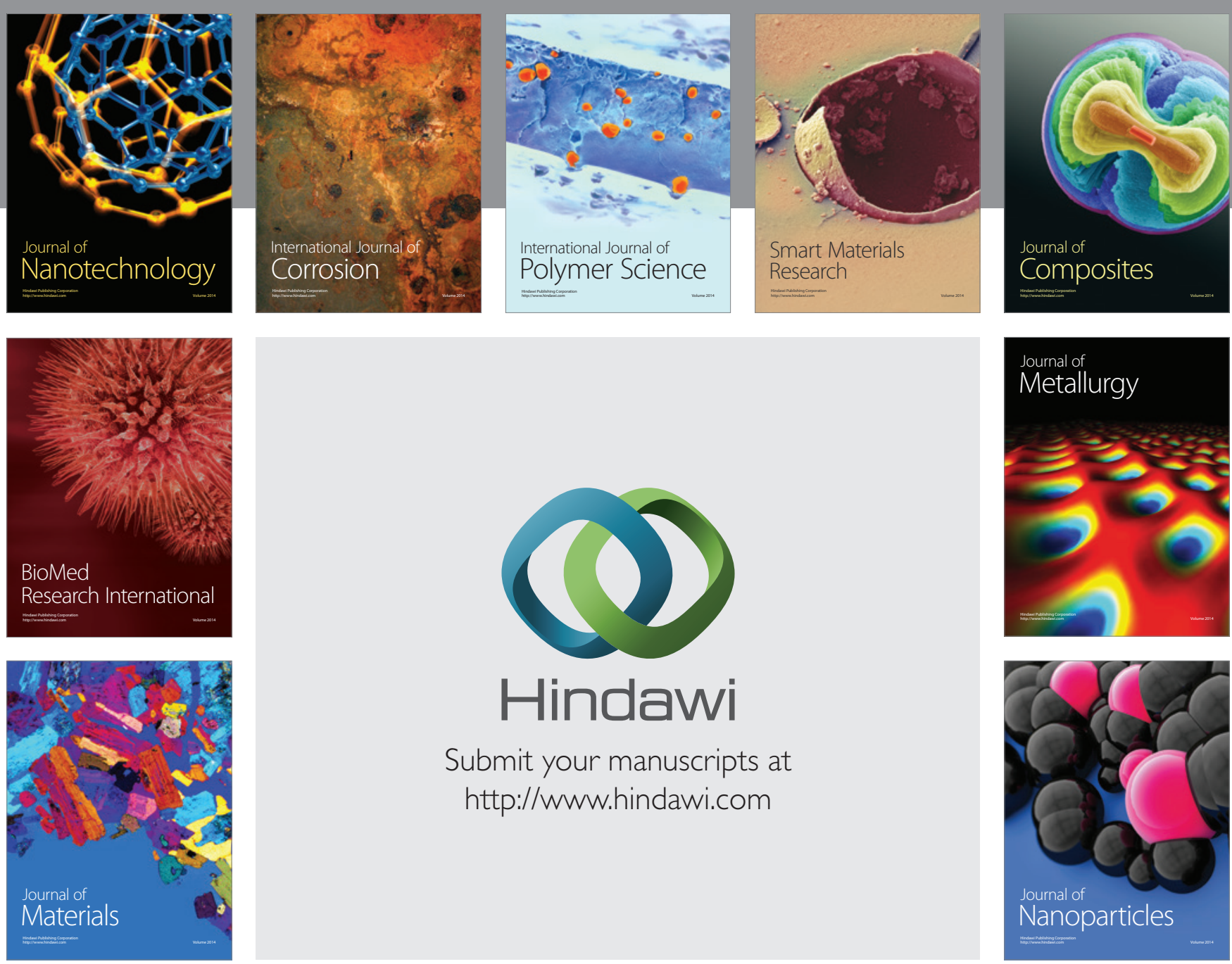

Submit your manuscripts at http://www.hindawi.com
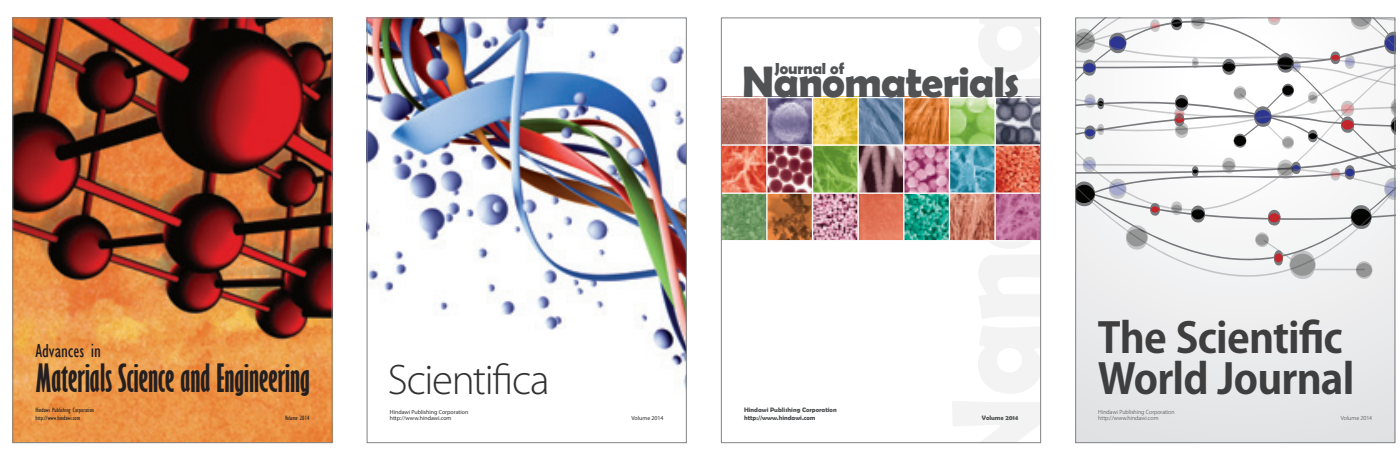

\section{The Scientific World Journal}
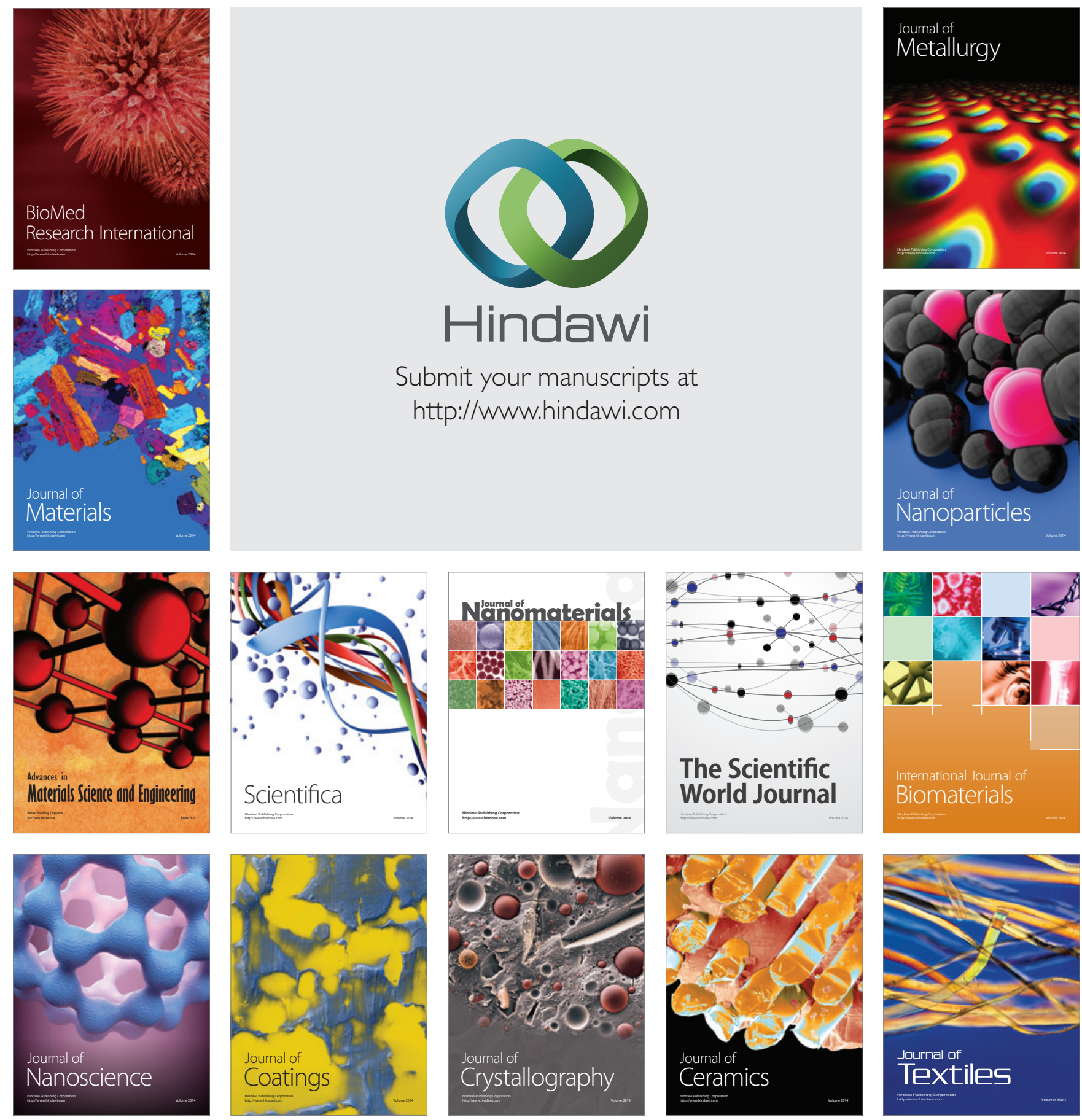